\title{
Neuroendocrine neoplasms of the digestive tract: incorporating the 2019 WHO grading schema in the South African context
}

\author{
AP Aldera, 1,2 ID D Govender, ${ }^{2,3}$ (D) ML Locketz ${ }^{2,4}$ iD \\ ${ }^{1}$ JDW Pathology Inc, South Africa \\ ${ }^{2}$ Division of Anatomical Pathology, University of Cape Town, South Africa \\ ${ }^{3}$ Pathcare, South Africa \\ ${ }^{4}$ National Health Laboratory Services, South Africa
}

Corresponding author,email: aldera@jdwpathology.co.za

Background: The classification of gastrointestinal (GI) neuroendocrine neoplasms (NENs) has been updated in the WHO classification of tumours of the digestive tract. Mitotic rate and Ki-67 proliferation index are central to the histopathological grading of these tumours. Assessing these variables is not standardised in local practice. This study addresses this deficit in the South African context.

Methods: This is a retrospective audit of archival material of resection specimens of GI NENs (excluding appendix) at Groote Schuur Hospital from 2004 to 2017. We performed a mitotic count and immunohistochemistry for Ki-67 to reclassify tumours according to the 2019 WHO criteria.

Results: Thirty-five resection specimens were examined. These represented stomach $(n=5)$, jejunoileum $(n=17)$, colon $(n=7)$ and rectum $(n=6)$. The mean age at the time of resection was 56 years (range 32-72). Twenty-one cases $(60 \%)$ were women and $14(40 \%)$ were men. The majority (24/35) of tumours were classified as grade 1 well-differentiated neuroendocrine tumours (WD NETs), 8/35 were classified as grade 2 WD NETs and 3/35 were classified as small cell poorly differentiated neuroendocrine carcinomas (PD NECs). Re-classification resulted in $7 / 35(20 \%)$ changes in tumour grade (4 upgraded, 3 downgraded). In most instances (7/8), the Ki-67 proliferation index was the determining factor in assigning tumour grade.

Conclusion: This study has increased awareness of the recently updated WHO classification system. We conclude that an objective and systematic approach to counting of mitoses and determination of the Ki-67 proliferation index is recommended in routine practice.

Keywords: pathology, neuroendocrine neoplasm, carcinoid, gastrointestinal pathology

\section{Introduction}

Neuroendocrine neoplasms (NENs) of the gastrointestinal (GI) tract are a heterogeneous group of tumours with diverse biologic and clinical behaviours that vary according to anatomical distribution, tumour grade and neuroendocrine cell type. ${ }^{1,2}$ Siegfried Oberndorfer was the first to describe carcinoid tumours of the terminal ileum in 1907. ${ }^{3}$ Since this original description, the classification of these relatively uncommon tumours has undergone several revisions. According to the Surveillance Epidemiology and End Results (SEER) database, the annual incidence of GI NENs in the United States is approximately 1 per 100000 population. ${ }^{4}$ The 2019 World Health Organization (WHO) classification of tumours of the digestive system broadly categorises NENs as well-differentiated neuroendocrine tumours (WD NETs) and poorly differentiated neuroendocrine carcinomas (PD NECs). ${ }^{5}$ This distinction is made on light microscopy and is based on the tumour morphology. WD NETs are further subclassified into different histological grades based on proliferation indices (Table I). One of the most significant updates in the 2019 WHO classification is the addition of the category of grade 3 WD NETs. These tumours have a high mitotic rate $\left(>20 / 2 \mathrm{~mm}^{2}\right)$ and/or Ki-67 proliferation index (> 20\%) but resemble WD NETs morphologically and behave in a less aggressive manner than PD NEC. 5,6

Tumour cell proliferation is one of the most important histological prognostic factors for WD NET. ${ }^{7}$ Grading of WD NETs is achieved by performing a mitotic count and determining the $\mathrm{Ki}-67$ proliferation index. ${ }^{5,8,9}$ There are

\begin{tabular}{lcc}
\hline \multicolumn{2}{c}{ Table I: The 2019 WHO classification of GI NENs } \\
\hline $\begin{array}{c}\text { Mitotic count } \\
\text { (per } \mathbf{2} \mathbf{~ m m}^{2} \text { ) }\end{array}$ & $\begin{array}{c}\text { Ki-67 proliferation } \\
\text { index }\end{array}$ \\
\hline Well-differentiated NET \\
Grade 1 & $<2$ & $<3 \%$ \\
Grade 2 & $2-20$ & $3-20 \%$ \\
Grade 3 & $>20$ & $>20 \%$ \\
Poorly differentiated NEC & \\
Small cell type & $>20$ & $>20 \%$ \\
Large cell type & $>20$ & $>20 \%$
\end{tabular}


several methods which can be used to achieve this and there is a lack of standardisation between pathologists and between institutions. It is currently recommended to count the mitotic figures in an area of $10 \mathrm{~mm}^{2}$ (50 fields at $40 \mathrm{x}$ magnification and an ocular field diameter of $0.5 \mathrm{~mm}$ ) and then report the rate as per $2 \mathrm{~mm}^{2} .5,10$ Determining the $\mathrm{Ki}$ 67 proliferation index is useful, particularly when dealing with small biopsy specimens where counting the required number of high-power fields is challenging. In general, there are three techniques used by pathologists to estimate the Ki67 proliferation index. These include the eyeball technique, manual counting and digital automated counting. The 2019 WHO guidelines advocate for the manual counting method which is achieved by identifying the region of highest immunolabelling (hotspot) and counting at least 500 cells in this region. ${ }^{5}$ There is, however, no universally accepted technique for performing the Ki-67 count. Most experts advocate a manual count, which can be done digitally or on a printed photomicrograph. ${ }^{11-14}$ Performing these labour intensive counts is time-consuming and not routinely done in practice. This study aimed to apply objective standardised techniques in evaluating the mitotic rate and Ki-67 proliferation index to GI NEN resections according to the latest (2019) WHO criteria and study their effect on tumour classification.

\section{Methods}

This was a retrospective study performed on archival material of cases seen at Groote Schuur Hospital (GSH) in the Division of Anatomical Pathology, National Health Laboratory Service and the University of Cape Town (UCT), South Africa. Patients who underwent excision of their NEN between 2003 and 2017 were included. The cases were identified by searching the laboratory information systems. Data were extracted from the histology reports. Variables recorded included patient age, sex, site of tumour and lymph node metastases. Cases with incomplete records and formalin-fixed paraffin-embedded (FFPE) tissue blocks which were unsuitable for further study were excluded. This study was limited to the GI tract from the stomach to the rectum and excluded pancreatic NENs. Appendicectomy cases were excluded from this study due to the small size of the tumours inherently present in these specimens which are not amenable to thorough mitotic counts.

The tissue block representing the largest proportion of tumour was selected for mitotic counts and immunohistochemistry. We performed a formal mitotic count on haematoxylin and eosin (H\&E) stained sections by identifying the area of the tumour with the most mitotic activity on scanning magnification (the so-called hotspot). The number of mitotic figures was then counted in an area of $10 \mathrm{~mm}^{2}$. The mitotic rate was then reported as per $2 \mathrm{~mm}^{2}$.
Ki-67 immunohistochemistry was performed using the MIB1 clone (1:100, Dako, Denmark) on all cases. The Ki67 proliferation index was determined by identifying the area with the highest number of positively staining tumour cells on scanning magnification (hotspot). This area was photographed at high magnification (400x). Using the Microsoft Paint software programme (Washington, USA), a red dot was placed over all the nuclei which showed positive labelling with the Ki-67 immunostain. Black dots were placed over the haematoxylin-stained tumour cell nuclei which did not label with the Ki-67 antibody until a total of 500 cells had been counted. Care was taken to only include tumour cells in this count, and lymphocytes, endothelial cells and fibroblasts were excluded. The Ki-67 proliferation index was expressed as a percentage.

For the purposes of this study we used the 2019 WHO 5 th edition classification schema for grading neuroendocrine neoplasms of the digestive system (Table I). In cases where there was discrepancy between the Ki-67 proliferation index and the mitotic count we assigned the higher tumour grade.

\section{Results}

Fifty-three cases were identified from the laboratory information system during the study period 2003-2017. Thirtyfive were retrieved from the archives that were suitable for further evaluation. The anatomic distribution of the tumours was 16 jejunoileum, seven colon, six rectum and five stomach cases (Table II). The mean age of the patients at the time of resection was 56 years (range 32-72). Twenty-one cases $(60 \%)$ were women and $14(40 \%)$ were men.

Twenty-four tumours $(68.5 \%)$ were classified as grade 1 WD NETs. These tumours occurred in the stomach $(n=3)$, jejunoileum $(n=12)$, colon $(n=4)$ and rectum $(n=5)$. The mean mitotic rate for the grade 1 WD NETs was 0.2 per 2 $\mathrm{mm}^{2}$ (range $0-1.2$ ) and the mean Ki-67 proliferation index was $1.2 \%$ (range $0.2-2.9 \%$ ). There were eight $(22.8 \%$ ) tumours that were classified as grade 2 WD NETs. These tumours occurred in the stomach $(n=1)$, jejunoileum $(n=5)$, colon $(n=1)$ and rectum $(n=1)$. The mean mitotic rate for the grade $2 \mathrm{WD}$ NETs was 0.7 per $2 \mathrm{~mm}^{2}$ (range $0-2.2$ ) and the mean Ki-67 proliferation index was $4.3 \%$ (range $0.6-6.8 \%$ ). The mitotic rate alone only defined one $(12.5 \%)$ tumour as a grade 2 WD NET; in the other seven cases, the grade defining marker of proliferation was the Ki-67 proliferation index. There were no tumours that met the criteria for grade 3 WD NET. There were three $(8.5 \%)$ tumours that were classified as small cell PD NECs. These tumours occurred in the stomach $(n=1)$ and colon $(n=2)$ only. The mean mitotic rate for the small cell PD NECs was 31.3 per $2 \mathrm{~mm}^{2}$ (range 24.2-43.2) and the mean Ki-67 proliferation index was $53.7 \%$ (range $40.7-84.4 \%$ ).

Table II: Summary of demographic information and reclassified tumour grades

\begin{tabular}{|c|c|c|c|c|c|c|}
\hline \multirow{2}{*}{ Site } & \multirow{2}{*}{ Age, mean, range (years) } & \multirow{2}{*}{ Male } & \multirow{2}{*}{ Female } & \multicolumn{2}{|c|}{ WD NET } & \multirow{2}{*}{$\begin{array}{c}\text { PD NEC } \\
\text { Small cell }\end{array}$} \\
\hline & & & & G1 & G2 & \\
\hline Stomach $(n=5)$ & $55.6(37-72)$ & 2 & 3 & 3 & 1 & 1 \\
\hline Jejunoileum $(n=17)$ & $54.4(32-70)$ & 8 & 9 & 12 & 5 & 0 \\
\hline Colon $(n=7)$ & $59.4(51-70)$ & 2 & 5 & 4 & 1 & 2 \\
\hline $\operatorname{Rectum}(n=6)$ & $57.5(37-76)$ & 2 & 4 & 5 & 1 & 0 \\
\hline Overall & & 14 & 21 & 24 & 8 & 3 \\
\hline
\end{tabular}




\begin{tabular}{|c|c|c|}
\hline & Positive & Negative \\
\hline Grade 1 WD NET & 12 & 8 \\
\hline Grade 2 WD NET & 5 & 1 \\
\hline Small cell PD NEC & 2 & 1 \\
\hline Total & 19 & 10 \\
\hline
\end{tabular}

In all three cases of PD NEC both the mitotic rate and Ki-67 proliferation index met the diagnostic criteria.

Seven tumours $(20 \%)$ were reclassified in this study. Four tumours that were originally diagnosed as "carcinoid tumours" or grade 1 WD NETs were reclassified as grade 2 WD NETs. These tumours were from the colon $(n=1)$ and small intestine $(n=3)$. The reclassification was based on the mitotic count $(n=1)$ or Ki-67 proliferation index $(n=3)$. In addition, three tumours that were originally classified as grade 2 WD NETs were reclassified as grade 1 WD NETs. These tumours were from the rectum $(n=1)$ and jejunoileum $(n=2)$. There were no changes in classification between WD NETs and PD NECs.

Twenty-nine tumour resections (82.9\%) included lymph node dissections (Table III). Nineteen of these cases $(65.5 \%)$ showed regional lymph node metastases. Grade 1 WD NETs showed lymph node metastases in $12 / 20$ cases $(60 \%)$, grade 2 WD NETs $5 / 6$ cases $(83.3 \%)$ and small cell PD NEC $2 / 3$ cases $(66.7 \%)$. Fisher's exact test revealed that these results were not statistically significant $(p=0.3798)$. Of the tumours that were downgraded from grade 2 WD NET to grade 1 WD NET, $1 / 3$ cases $(33.3 \%)$ demonstrated lymph node metastases. Tumours which were upgraded from grade 1 WD NET to grade 2 WD NET showed lymph node metastases in $2 / 3$ cases $(66.7 \%)$. One of the tumours which had a change in grade did not have a lymph node dissection.

\section{Discussion}

The terminology and classification surrounding NENs of the GI tract are confusing and have undergone several revisions. The term "carcinoid" has been used to describe most GI NETs since 1980 when it was proposed by the WHO and has become entrenched in the medical literature. However, the term does not adequately convey the malignant potential that these tumours often exhibit. ${ }^{1}$ The $2000 \mathrm{WHO}$ classification system divided GI NENs into WD NETs and PD NECs based on the degree of differentiation. WD NETs were further classified based on mitotic count and Ki-67 proliferation index. WD NETs were regarded as low-grade malignancies and PD NECs were considered high-grade malignant tumours. The 2010 WHO classification categorised all GI NENs as malignant tumours, except for pancreatic neuroendocrine microadenomas, L-cell-type NETs and tubular carcinoids. In the 2010 WHO classification system, NENs were divided into grade 1 NETs, grade 2 NETs and grade 3 NECs based on mitotic count and Ki-67 proliferation index. The cut off values used for mitoses and Ki-67 are the same in the 2010 and 2019 WHO classification systems. The 2019 WHO classification has separated tumours designated by the 2010 system as "grade 3 NECs" into grade 3 WD NETs and PD NECs based on tumour morphology. ${ }^{6}$

The accurate grading of NENs of the digestive tract is important for prognostication, follow-up and optimal patient management. ${ }^{15}$ In this study, we have demonstrated that $7 / 35$ tumours $(20 \%)$ were reclassified when the mitotic rate and $\mathrm{Ki}-67$ were evaluated in a meticulous and systematic manner. This is supported by several larger studies published in international literature. ${ }^{11-14}$ This study also highlights that there can be a discrepancy between the mitotic rate and Ki-67 proliferation index, and that the higher of these two indices should be used for grading. ${ }^{9}$ This could be due to preanalytical variables such as the ischaemic time, which has been shown to affect the mitotic count. ${ }^{16}$

Interestingly, there were no grade $3 \mathrm{WD}$ NETs in this cohort of excision specimens. It is likely that in the past, due to the high Ki-67 proliferation index that these tumours exhibit, these tumours would have been classified as small cell PD NECs and would not have been resected. This highlights the importance of distinguishing grade 3 WD NETs from PD NECs because there are significant differences in prognosis. ${ }^{6,17}$

WD NETs are well known to exhibit heterogeneity and show different proliferation rates in different areas of the tumour. ${ }^{5}$ This is overcome somewhat by the recommendation of counting mitoses in an area of $10 \mathrm{~mm}^{2}$ and then reporting the rate as per $2 \mathrm{~mm}^{2}$. Selecting the tumour proliferation "hotspot" on intermediate power and then counting 500 rather than 100 tumour cells allows for the most proliferative region of the tumour to be used for grading.

Current practice is to perform $\mathrm{Ki}-67 \mathrm{immunohistochemistry}$ on the primary tumour as well as any metastases and to assign the tumour the higher grade for treatment purposes. Dhall et al. showed that a Ki-67 proliferation index of more than $2 \%$ at either the primary or a metastatic site was the only significant predictor of progression-free survival in a cohort of 57 ileal NETs. ${ }^{18}$ These findings have been supported by a more recent study by Shi et al. who examined a cohort of 27 small bowel NETs in which liver metastases had been resected. ${ }^{19}$ In this study, all of the primary tumours were grade $1 / 2$ NETs, but eight patients $(30 \%)$ were found to have a grade 3 liver metastasis. Progression-free survival in these patients was 7 months versus 38 months for patients without a G3 liver metastasis.

In the staging system of The American Joint Committee on Cancer (AJCC), NETs of the stomach, small intestine, and colorectum have specially designated staging systems. Yao et al. performed a meta-analysis on SEER data, and found that the proportion of GI NET cases presenting with lymph node metastases varied by anatomic site. ${ }^{20}$ The caecum was the site associated with the highest proportion of lymph node metastases (42\%), followed by jejunum/ileum (41\%), appendix (28\%), colon (23\%), duodenum (10\%), stomach $(9 \%)$, and rectum $(4 \%){ }^{20}$ This study did not stratify the cases with lymph node metastases by histological grade. In our study, we found lymph node metastases in $60 \%$ of grade 1 WD NETs and in $83.3 \%$ of grade 2 WD NETs. These results indicate that higher grade WD NETs are more likely to present with lymph node metastases, but these results were not statistically significant.

The results of this study were not statistically significant due to the small sample size. GI NETs are uncommon tumours, and we only identified 53 resection specimens over the 15 -year study period. We decided to limit our study to resection specimens in order to evaluate the mitotic rate over an area of $10 \mathrm{~mm}^{2}$ and to evaluate the lymph node status. Although the appendix is the most common site for GI 
NENs, these specimens were excluded from our study. Appendiceal NENs are often small and unsuitable for performing a formal mitotic count over an area of $10 \mathrm{~mm}^{2}$. In fact, they are often so small that they get cut away when performing additional stains. Additionally, there would be no information on record about metastatic disease as lymph nodes would not have been removed in the majority of these tumours.

\section{Conclusion}

We have described the demographic variables of these tumours in a South African setting. We have demonstrated that the technique of counting mitotic figures in an area of $10 \mathrm{~mm}^{2}$ and objectively evaluating the Ki-67 proliferation index with manual counting of a digital photomicrograph resulted in reclassification of $20 \%$ of tumours. We therefore recommend these techniques be used in routine practice, along with the new 2019 WHO classification system for GI NENs and linked to clinical outcomes to assess the prognostic value of these changes.

\section{Conflict of interest}

The authors declare no conflict of interest.

\section{Funding source}

Funding for this study was obtained from the National Health Laboratory Service (NHLS) Research Trust Development Grant (Reference number: 2018-1DEV21-MLO01).

\section{Ethical approval}

Ethical approval for this study was obtained from the University of Cape Town Faculty of Health Sciences Human Research Ethics Committee (Reference number: 176/2018).

\section{ORCID}

AP Aldera (iD https://orcid.org/0000-0002-9615-1692 D Govender (iD https://orcid.org/0000-0003-1487-8255 ML Locketz iD https://orcid.org/0000-0002-3917-3895

\section{REFERENCES}

1. Klimstra DS, Modlin IR, Coppola D, Lloyd RV, Suster S. The pathologic classification of neuroendocrine tumors: a review of nomenclature, grading, and staging systems. Pancreas. 2010;39(6):707-12.

2. Klöppel G, Couvelard A, Perren A, et al. ENETS consensus guidelines for the standards of care in neuroendocrine tumors: towards a standardised approach to the diagnosis of gastroenteropancreatic neuroendocrine tumors and their prognostic stratification. Neuroendocrinol. 2009;90(2):162-6. $400 x$ )

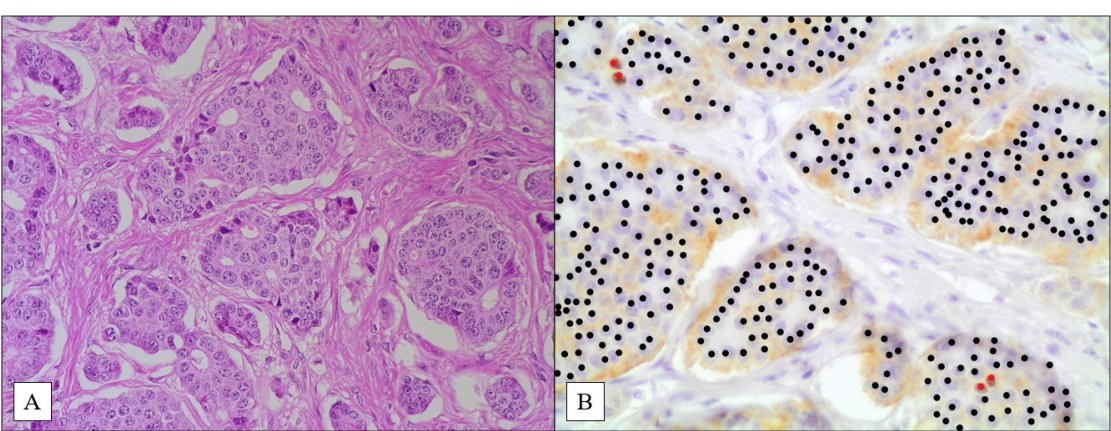

Figure 1: Grade 1 WD NET

A. Nests of uniform neuroendocrine cells with an insular growth pattern (H\&E,

B. Illustration of the digital manual counting technique for the Ki-67 of this grade 1 WD NET (400x)

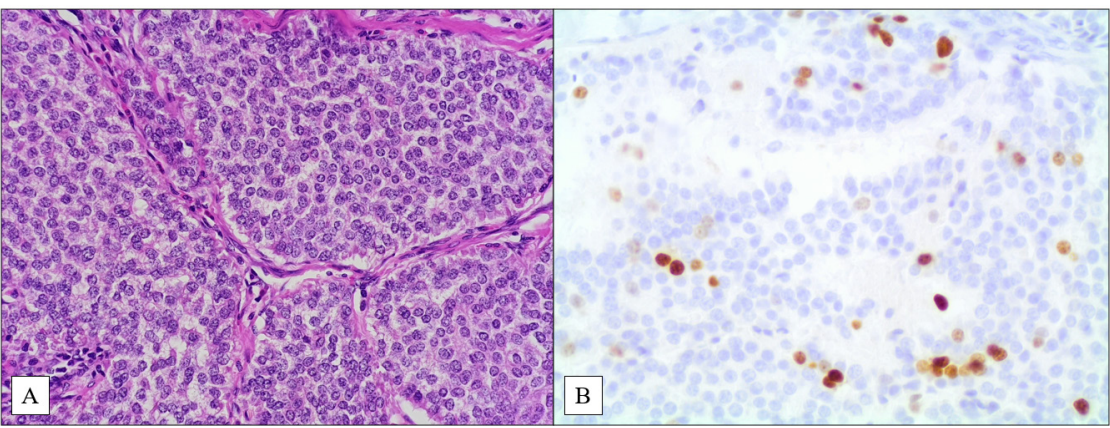

Figure 2: Grade 2 WD NET

A. Sheets of neuroendocrine cells with low nuclear-to-cytoplasmic ratios and typical stippled chromatin (H\&E, 400x)

B. Ki-67 immunohistochemistry before digital manual counting demonstrating (1)

Figure 3: Small cell PD NEC

A. Sheets of malignant cells with high nuclear-to-cytoplasmic ratios and numerous mitotic figures ( $H \& E$, 400x)

Illustration of the digital manual counting technique demonstrating a Ki-67 proliferative index of $35.9 \%(400 x)$

3. Modlin IM, Shapiro MD, Kidd M, Eick G. Siegfried Oberndorfer and the evolution of carcinoid disease. Arch Surg. 2007;142(2):187-97.

4. Dasari A, Shen C, Halperin D, et al. Trends in the incidence, prevalence, and survival outcomes in patients with neuroendocrine tumors in the United States. JAMA Oncology. 2017;3(10):1335-42.

5. WHO Classification of Tumours Editorial Board. Digestive system tumours. 5th ed. Lyon (France): International Agency for Research on Cancer; 2019.

6. Tang LH, Untch BR, Reidy DL, et al. Well-differentiated neuroendocrine tumors with a morphologically apparent high-grade component: a pathway distinct from poorly differentiated neuroendocrine carcinomas. Clin Cancer Res. 2016;22(4):1011-7. 
7. Jamali M, Chetty R. Predicting prognosis in gastroenteropancreatic neuroendocrine tumors: an overview and the value of Ki-67 immunostaining. Endocr Pathol. 2008;19(4):282-8.

8. Klöppel G, Rindi G, Perren A, Komminoth P, Klimstra DS. The ENETS and AJCC/UICC TNM classifications of the neuroendocrine tumors of the gastrointestinal tract and the pancreas: a statement. Virchows Archiv. 2010;456(6):595-7.

9. Van Velthuysen M-LF, Groen EJ, Van Der Noort V, et al. Grading of neuroendocrine neoplasms: mitoses and Ki-67 are both essential. Neuroendocrinol. 2014;100(2-3):221-7.

10. Amin MB, Edge S, Greene FL, et al, editors. AJCC Cancer Staging Manual. 8th ed. New York, NY: Springer; 2017.

11. Bagci P, Ohike N, Dursun N, et al. Comparative analysis of different counting methodologies for Ki-67 in pancreatic neuroendocrine tumors (abstract). Mod Pathol. 2012;25:441A.

12. Tang LH, Gonen M, Hedvat C, Modlin IM, Klimstra DS. Objective quantification of the Ki-67 proliferative index in neuroendocrine tumors of the gastroenteropancreatic system: a comparison of digital image analysis with manual methods. Am J Surg Pathol. 2012;36(12):1761-70.

13. Kroneman TN, Voss JS, Lohse CM, et al. Comparison of three Ki-67 index quantification methods and clinical significance in pancreatic neuroendocrine tumors. Endocr Pathol. 2015;26(3):255-62.

14. Reid MD, Bagci P, Ohike N, et al. Calculation of the Ki-67 index in pancreatic neuroendocrine tumors: a comparative analysis of four counting methodologies. Mod Pathol. 2015;28(5):686.

15. Rorstad O. Prognostic indicators for carcinoid neuroendocrine tumors of the gastrointestinal tract. J Surg Oncol. 2005;89(3):151-60.

16. Cross S, Start R, Smith J. Does delay in fixation affect the number of mitotic figures in processed tissue? J Clin Pathol. 1990;43(7):597-9.

17. Sorbye H, Welin S, Langer SW, et al. Predictive and prognostic factors for treatment and survival in 305 patients with advanced gastrointestinal neuroendocrine carcinoma (WHO G3): the NORDIC NEC study. Ann Oncol. 2013;24(1):15260

18. Dhall D, Mertens R, Bresee C, et al. Ki-67 proliferative index predicts progression-free survival of patients with well-differentiated ileal neuroendocrine tumors. Hum Pathol. 2012;43(4):489-95.

19. Shi C, Gonzalez RS, Zhao Z, et al. Liver metastases of small intestine neuroendocrine tumors: Ki-67 heterogeneity and World Health Organization grade discordance with primary tumors. Am J Clin Pathol. 2015;143(3):398-404.

20. Yao JC, Hassan M, Phan A, et al. One hundred years after "carcinoid": epidemiology of and prognostic factors for neuroendocrine tumors in 35,825 cases in the United States. J Clin Oncol. 2008;26(18):3063-72. 